\title{
XELIRI regimen plus continuous treatment with bevacizumab is well-tolerated and effective in metastatic colorectal cancer patients in a second-line setting involving the sequential administration of XELOX and XELIRI
}

\author{
KOICHI SUZUKI ${ }^{1}$, KATO TAKAHARU $^{1}$, YUTA MUTO ${ }^{1}$, KOSUKE ICHIDA $^{1}$, TARO FUKUI ${ }^{1}$, \\ YUJI TAKAYAMA ${ }^{1}$, SHINGO TSUJINAKA ${ }^{1}$, JUNICHI SASAKI ${ }^{1}$, HISANAGA HORIE ${ }^{1}$, \\ YUTAKA J. KAWAMURA ${ }^{1}$, FUMIO KONISHI ${ }^{2}$ and TOSHIKI RIKIYAMA ${ }^{1}$ \\ ${ }^{1}$ Department of Surgery, Saitama Medical Center, Jichi Medical University, Saitama 330-8503; \\ ${ }^{2}$ Nerima-Hikarigaoka Hospital, Tokyo 179-0072, Japan
}

Received February 12, 2014; Accepted May 26, 2014

DOI: $10.3892 / \mathrm{mco} .2014 .306$

\begin{abstract}
The aim of the present study was to present a retrospective review of 42 metastatic colorectal cancer (mCRC) patients treated using the XELIRI regimen as second-line chemotherapy during the period between 2010 and 2012 . Patients were treated with capecitabine, 1,600 ( $\geq 65$ years) or $2,000 \mathrm{mg} / \mathrm{m}^{2}$ ( $<65$ years), on days $1-15,200 \mathrm{mg} / \mathrm{m}^{2}$ irinotecan (CPT-11) on day 1, with or without $7.5 \mathrm{mg} / \mathrm{kg}$ bevacizumab on day 1 and every 21 days. A total of 21 patients underwent XELIRI and 21 underwent XELIRI plus bevacizumab treatment. Fifteen patients received continuous administration of bevacizumab in the first- and second-line settings [bevacizumab beyond progression $(\mathrm{BBP})+]$, whereas 27 patients did not receive the treatment (BBP-). Forty patients (95.2\%), including all the patients in the $\mathrm{BBP}+$ group, received sequentially administered XELOX and XELIRI regimens from the first- to the second-line setting. The disease control rate (DCR), progression-free survival (PFS), overall survival (OS) and adverse events were compared between the BBP- and BBP+ groups. The median relative dose intensity was similar (93.9\% for capecitabine and $96.3 \%$ for CPT-11 in the BBP- group vs. $94.8 \%$ for capecitabine and $91.5 \%$ for CPT-11 in the BBP+ group). The DCR was $25.9 \%$ in the BBPand $66.6 \%$ in the $\mathrm{BBP}+$ groups $(\mathrm{P}=0.020)$. The median PFS was 3.5 months in the BBP- and 7.2 months in the BBP+ groups $(\mathrm{P}=0.028)$. The $\mathrm{BBP}+$ group exhibited a higher median $\mathrm{OS}$ time compared to the BBP-group (12.5 months in the BBP- group vs. not reached in the $\mathrm{BBP}+$ group; $\mathrm{P}=0.0267)$. The most common grade $3 / 4$ adverse event $(n \geq 20)$ was hypertension observed in
\end{abstract}

Correspondence to: Dr Koichi Suzuki, Department of Surgery, Saitama Medical Center, Jichi Medical University, 1-847 Amanuma-cho, Omiya-ku, Saitama 330-8503, Japan

E-mail:ksuzbnhm@yahoo.co.jp

Key words: XELOX, XELIRI, bevacizumab, capecitabine, irinotecan, metastatic colorectal cancer the BBP+ group [three patients (20\%)]: these three patients were well-controlled with a single antihypertensive drug. Treatment with sequentially administered XELOX and XELIRI regimens did not aggravate adverse events in the 40 patients. The results showed that the XELIRI regimen, involving continuous treatment with bevacizumab, was well-tolerated and effective as a second-line chemotherapy and sequentially administering XELOX and XELIRI was feasible and manageable for patients with $\mathrm{mCRC}$.

\section{Introduction}

Capecitabine is widely used in combination with oxaliplatin (XELOX regimen) in the treatment of metastatic colorectal cancer (mCRC). Treatment with bevacizumab in addition to XELOX has been shown to be effective and well-tolerated in patients with mCRC in the NO16966 (1) and TREE2 (2) clinical trials. These studies showed that the XELOX regimen had the similar benefit in progression-free survival (PFS) and overall survival (OS) as compared with the standard FOLFOX regiment (intraneous infusion of flurouracil/folinic acid plus oxaliplatin). The addition of bovacizumab to XELOX regimen significantly improved PFS and showed a similar toxicity profile compared to those documented in previous studies $(1,2)$.

The combination of irinotecan (CPT-11) with capecitabine (XELIRI regimen) with or without bevacizumab has also been shown to be a well-tolerated and effective regimen in the treatment of $\mathrm{mCRC}$ in a first-line setting. The XELIRI and XELOX regimens are more convenient for the patient as they do not require the use of a home-infusion pump, and as a result of the tri-weekly schedule, the number and duration of infusion visits are also reduced. Sequential administration of XELOX and XELIRI in a first- to second-line setting would allow patients to be managed more easily in an outpatient unit. Furthermore, continuous treatment with bevacizumab plus XELOX and XELIRI may provide clinical benefits as shown in the ML18147 study (3) involving patients with mCRC subsequent to their first progression. However, only a small number 
of studies have addressed the benefits of sequential administration of XELOX and XELIRI plus bevacizumab and concerns regarding cumulative adverse events as a consequence of the continuous use of bevacizumab and capecitabine remain.

In the present study, the efficacy and safety of the XELIRI regimen was evaluated in combination with continuous treatment using bevacizumab for patients with $\mathrm{mCRC}$ in a second-line chemotherapy setting and the feasibility of a regimen involving the sequential administration of XELOX and XELIRI was elucidated.

\section{Materials and methods}

Patients and treatment. A total of 42 patients with mCRC were recruited in the present study. Patients had histologically confirmed and measurable mCRC and were treated in Jichi Medical University Saitama Medical Center between 2010 and 2012 using the XELIRI regimen as a second-line chemotherapy, following previous treatment involving standard first-line chemotherapy consisting of fluoropyrimidine plus oxaliplatin, with or without bevacizumab. CPT-11 $200 \mathrm{mg} / \mathrm{m}^{2}$ was administered on day 1 in combination with oral capecitabine, 1,600 (patients aged $\geq 65$ years) or $2,000 \mathrm{mg} / \mathrm{m}^{2}$ (patients aged $<65$ years), on days $1-15$, with or without $7.5 \mathrm{mg} / \mathrm{kg}$ bevacizumab on day 1 and q21 days.

Efficacy and safety assessment. The patients that received continuous administration of bevacizumab in the first- and second-line settings [bevacizumab beyond progression $(\mathrm{BBP}+)]$ and those that did not (BBP-) were compared regarding the assessment of the disease control rate (DCR), PFS, OS and adverse events. Tumors were evaluated following every 3 or 4 cycles of chemotherapy using a computed tomography scan and other tests that were initially used to stage the tumor. Tumor response and progression were evaluated according to the Response Evaluation Criteria In Solid Tumors. Adverse events were graded according to the Common Terminology Criteria for Adverse Events, version 3.0. Treatment was continued until disease progression, unacceptable toxicity, deterioration of Eastern Cooperative Oncology Group performance status to $>2$ or withdrawal of patient consent. Written informed consent was obtained from all patients prior to receiving chemotherapy, according to the Jichi Medical University Institutional Review Board.

KRAS mutation analysis. KRAS status was evaluated by means of the Scorpion amplified refractory mutation system method using formalin-fixed paraffin-embedded tumor tissue from patients subsequent to obtaining informed consent. KRAS analysis was performed by a clinical testing company (Special Reference Laboratories, Inc., Tokyo, Japan).

Statistical analysis. Fisher's exact test was used to examine the association between two categorical variables. Continuous comparisons of variables between two groups were performed: The Student's t-test was used to determine those variables that followed a normal distribution, while the non-parametric Mann-Whitney U test was used for those variables that did not follow a normal distribution. $\mathrm{P}<0.05$ was considered to indicate a statistically significant difference. Values are shown
Table I. Patient characteristics.

\begin{tabular}{lcc}
\hline Characteristics & BBP- $(\mathrm{n}=27)$ & $\mathrm{BBP}+(\mathrm{n}=15)$ \\
\hline Age, years & & \\
Median (range) & $65(37-85)$ & $67(47-83)$ \\
Gender, $\mathrm{n}$ & 21 & 9 \\
Male & 6 & 6 \\
Female & & \\
Primary tumor site, $\mathrm{n}$ & 15 & 5 \\
Colon & 12 & 10 \\
Rectum & & \\
Resection of primary tumor, $\mathrm{n}$ & 24 & 15 \\
Yes & & \\
Metastatic site(s), $\mathrm{n}$ & 13 & 10 \\
Liver & 9 & 8 \\
Lung & 4 & 5 \\
Lymph nodes & 6 & 3 \\
Peritoneum & 10 &
\end{tabular}

BBP, bevacizumab beyond progression.

as the mean \pm standard error. PFS and OS data were plotted as Kaplan-Meier curves and the differences among the groups were compared using the log-rank test.

\section{Results}

Patient characteristics. A total of 21 patients underwent treatment with XELIRI and 21 underwent XELIRI with bevacizumab. In total 15 patients received continuous administration of bevacizumab in the first- and second-line settings (BBP+), whereas 27 did not receive bevacizumab (BBP-). The BBP-group included patients treated with bevacizumab in either the first- or second-line settings. All the 15 patients in the $\mathrm{BBP}+$ group received a regimen involving sequential administration of XELOX and XELIRI from the first- to the second-line settings. Among the 27 patients in the BBP-group, 25 received sequential administration of XELOX and XELIRI and the other two received mFOLFOX6 (patients were treated with $200 \mathrm{mg} / \mathrm{m}^{2}$ folinic acid, $400 \mathrm{mg} / \mathrm{m}^{2}$ fluorouracil (5-FU) and $85 \mathrm{mg} / \mathrm{m}^{2}$ oxaliplatin on day 1 , followed by a $46-\mathrm{h}$ continuous infusion of $2,400 \mathrm{mg} / \mathrm{m}^{2} 5-\mathrm{FU}$ on days 1 and 2) in addition to the XELIRI regimen (Fig. 1). No significant differences in characteristics were found between the BBP- and $\mathrm{BBP}+$ groups in terms of median age, gender, primary tumor site, resection of primary tumor and metastatic site. Patient characteristics are shown in Table I.

Initial treatments. A total of 40 patients $(95 \%)$ underwent the XELOX regimen and two underwent the mFOLFOX6 regimen. Among the 40 patients treated with the XELOX regimen, 15 also received bevacizumab (Fig. 1). In the XELOX regimen, patients were treated with $2,000 \mathrm{mg} / \mathrm{m}^{2}$ capecitabine on days $1-15$ and $130 \mathrm{mg} / \mathrm{m}^{2}$ oxaliplatin on day 1 , with or without 
Table II. Initial treatment.

\begin{tabular}{lcc}
\hline Initial treatment & BBP- $(\mathrm{n}=27)$ & $\mathrm{BBP}+(\mathrm{n}=15)$ \\
\hline Regimen, $\mathrm{n}$ & & 0 \\
mFOLFOX6 $\pm \mathrm{BV}$ & 25 & 15 \\
XELOX \pm BV & & \\
Follow-up duration, months & $19.9(4.0-46.6)$ & $16.1(5.1-39.0)$ \\
Median (range) & & \\
Best overall response, $\mathrm{n}$ & 0 & 0 \\
Complete response & 0 & 0 \\
Partial response & 15 & 41 \\
Stable disease & 5 & 0 \\
Progressive disease & 7 & 8 \\
Not examined & & 3 \\
Reason for discontinuation, $\mathrm{n}$ & 16 & 2 \\
Progressive disease & 4 & 2 \\
Adverse events & 6 & \\
Allergy & 1 & \\
Others & & \\
\hline
\end{tabular}

BBP, bevacizumab beyond progression; BV, bevacizumab.

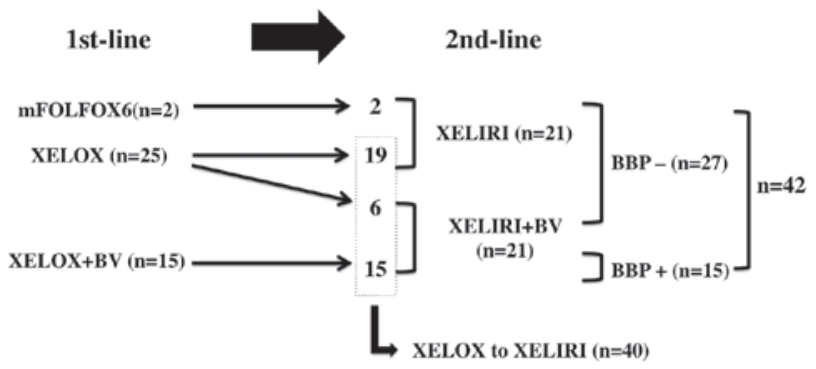

Figure 1. Treatments for patients in the first- and second-line settings Bevacizumab beyond progression $(\mathrm{BBP})+$, patients who received continuous administration of bevacizumab in the first- and second-line settings. BBP-, patients who did not receive continuous administration of bevacizumab. BBP- includes patients treated with bevacizumab in either the first- or second-line settings. BV, bevacizumab.

$7.5 \mathrm{mg} / \mathrm{kg}$ bevacizumab on day 1 and q21 days. The median follow-up duration was 19.9 months [interquartile range (IQR), 4.0-46.6 months] in the BBP-group and 16.1 months (IQR, 5.1-39.0 months) in the BBP+ group. The most common reason for discontinuation of treatment was disease progression in each group. Initial treatments are summarized in Table II.

Treatment exposure. The median follow-up time was 10.2 months (IQR, 1.6-27.8 months) in the BBP-group and 10.2 months (IQR, 1.0-23.6 months) in BBP+ group. A total of 118 (median, 7.7 cycles) and 116 (median, 3.9 cycles) were administered in the BBP- and $\mathrm{BBP}+$ groups, respectively. Dose reductions were required for nine patients (33.3) in the $\mathrm{BBP}$ - group and six patients $(40 \%)$ in the $\mathrm{BBP}+$ group. The median relative dose intensities were $93.6 \%$ for capecitabine and $96.3 \%$ for CTP-11 in the BBP-group, and $94.8 \%$ for capecitabine and $91.5 \%$ for CTP-11 in the BBP+ group. No
Table III. Dose intensity.

\begin{tabular}{|c|c|c|c|c|}
\hline \multirow{2}{*}{$\begin{array}{l}\text { Dose } \\
\text { intensity }\end{array}$} & \multicolumn{2}{|c|}{ BBP- $(n=27)$} & \multicolumn{2}{|c|}{$\mathrm{BBP}+(\mathrm{n}=15)$} \\
\hline & Capecitabine & Irinotecan & Capecitabine & Irinotecan \\
\hline Relative, $\%$ & 93.9 & 96.3 & 94.8 & 91.5 \\
\hline Full dose, $n$ & 18 & 19 & 9 & 9 \\
\hline
\end{tabular}

$\mathrm{BBP}$, bevacizumab beyond progression.

Table IV. Response rate.

\begin{tabular}{lccccccc}
\hline Response rate & CR & PR & SD & PD & NE & RR & DCR \\
\hline BBP- $(\mathrm{n}=27), \mathrm{n}$ & 0 & 0 & 7 & 8 & 12 & 0 & 7 \\
$\%$ & 0 & 0 & 25.9 & 29.6 & 44.4 & 0 & 25.9 \\
$\mathrm{BBP}+(\mathrm{n}=15), \mathrm{n}$ & 1 & 0 & 9 & 2 & 3 & 1 & 10 \\
$\%$ & 6.7 & 0 & 60 & 13.3 & 20 & 6.7 & 66.7 \\
\hline
\end{tabular}

$\mathrm{CR}$, complete response; $\mathrm{PR}$, partial response; $\mathrm{SD}$, stable disease; $\mathrm{PD}$, progressive disease; NE, not examined; RR, response rate; DCR, disease control rate; BBP, bevacizumab beyond progression.

significant difference in relative dose intensity was found in any group (Table III).

Efficacy. One patient in the BBP+ group showed a complete response (CR), but there was no patient with a $\mathrm{CR}$ in the BBP- group. There were no patients observed with a partial response (PR) in either the $\mathrm{BBP}-$ or $\mathrm{BBP}+$ groups. A total of seven patients had stable disease (SD) in the BBP-group and 10 had SD in the BBP+ group. The BBP+ group exhibited a significantly improved DCR $(\mathrm{CR}+\mathrm{PR}+\mathrm{SD})$ compared with the BBP- group (25.9\% in the BBP- vs. $66.6 \%$ in the BBP+ groups; $\mathrm{P}=0.020$; Table IV). The median PFS was 3.5 months in the $\mathrm{BBP}$ - group and 7.2 months in the $\mathrm{BBP}+$ group $(\mathrm{P}=0.028$; Fig. 2A). The $\mathrm{BBP}+$ group also had a higher median OS time compared to the BBP-group (12.5 months in BBP-group vs. not reached in $\mathrm{BBP}+$ group; $\mathrm{P}=0.0267$; Fig. $2 \mathrm{~B})$. The hazard ratio between the $\mathrm{BBP}+$ and $\mathrm{BBP}$ - groups was 0.36 [95\% confidence interval (CI), 0.18-0.74] for PFS and 0.25 (95\% CI, 0.057-1.11) for OS. The PFS was compared between patients that underwent XELIRI with bevacizumab and XELIRI without bevacizumab, to elucidate the efficacy of the additional use of bevacizumab with XELIRI in the second-line setting. Regardless of the regimen used in the first-line setting, XELIRI with bevacizumab demonstrated an improved PFS as compared to XELIRI without bevacizumab in the second-line setting (3.7 months in BBP- vs. 6.3 months in the $\mathrm{BBP}+$ groups; $\mathrm{P}=0.0101$; Fig. $3 \mathrm{~A}$ ).

The ML18147 trial has demonstrated the benefits of continued use of bevacizumab plus standard second-line chemotherapy following standard first-line bevacizumab-based treatment (3). The criteria used in the ML18147 study excluded patients who were less likely to respond to first-line treatment, including those with a first-line PFS time of $<3$ months. In the current study, whether the patients with 

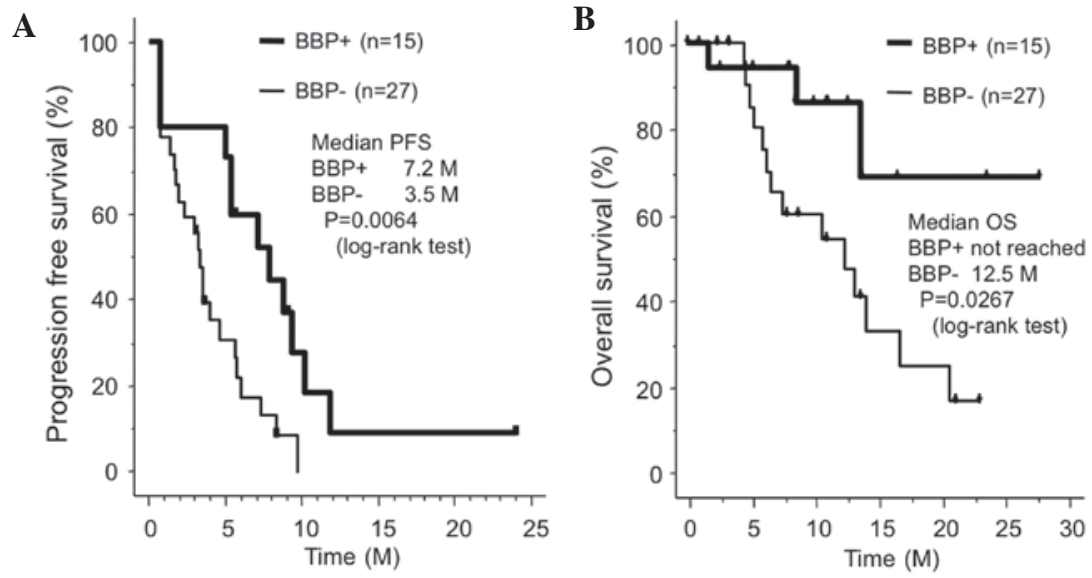

Figure 2. (A) Progression-free survival (PFS) and (B) overall survival (OS) rates according to the treatment regimen. BBP, bevacizumab beyond progression; M, months.
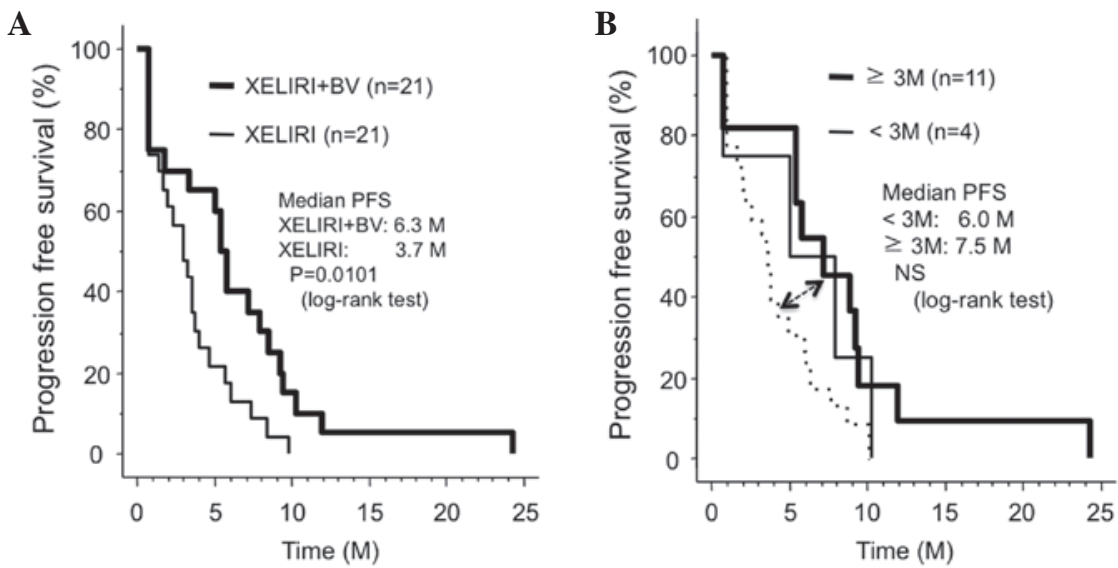

Figure 3. Comparison of second-line progression-free survival (PFS) time (A) between XELIRI and XELIRI + bevacizumab (BV) and (B) second-line PFS in the BBP+ group between patients with a first-line PFS time of $\geq 3$ months (M) and patients with a first-line PFS time $<3 \mathrm{M}$. Any patients in the BBP+ group, regardless of their response in a first-line setting, showed a higher second-line PFS than patients in the BBP-group (broken line). NS, no significance.

a poor response in the first-line setting showed a worse PFS time in the second-line setting than the other patients was evaluated. As long as continuous use of bevacizumab was maintained in the second-line setting, no difference in the second-line PFS time was found between patients with a poor response in the first-line setting and the other patients. Any patients in the BBP+ group, regardless of their response in the first-line setting, showed an improved second-line PFS time in association with the BBP- group [6.0 months in patients with a first-line PFS time of $<3$ months $(<3 \mathrm{M})$ and 7.5 months in first-line PFS time of $\geq 3$ months ( $\geq 3 \mathrm{M}$ ) vs. 3.5 months in the BBP-group; Fig. 3B].

Safety. The incidence of neutropenia [seven patients (22.2\%) in the BBP- group vs. nine patients $(60.0 \%)$ in the BBP+ group; $\mathrm{P}=0.007$ ] and hypertension [three patients (11.1) in the BBP- group vs. three patients $(53.3 \%)$ in the $\mathrm{BBP}+$ group; $\mathrm{P}=0.029]$ were significantly higher in $\mathrm{BBP}+$ compared to the BBP- group, but these adverse events were less than grade 3 . The most common grade 3 or higher adverse event $(\geq 20 \%)$ was hypertension observed in the BBP+ group [three patients (53.3\%), Table V]. All three patients in the BBP+ group exhibited grade 3 hypertension and were well controlled using a single antihypertensive drug. In the $\mathrm{BBP}+$ group, grade 4 neutropenia was found in one patient who was double heterozygous for the enzyme associated with the metabolism of CTP-11, i.e., uridine diphosphate-glucuronosyltransferase 1A1 (UGT1A1); however, the dose of CTP-11 was not reduced. No treatment-related mortalities were reported. The majority of the patients recovered from the aforementioned adverse events by reduction or discontinuation of the treatment. Treatment discontinuation due to disease progression occurred in 11 patients in the BBP- group (40.7\%) and nine patients in the BBP+ group $(60.0 \%)$. Three patients in the BBP- group (11.1) and three patients $(20 \%)$ in the BBP+ group treatment discontinued as a result of adverse events. Data regarding treatment discontinuation are summarized in Table VI.

KRAS status and subsequent therapies. KRAS mutation was found in three patients (11.1\%) in the BBP- and in three patients (20\%) in the BBP+ groups. A total of seven patients with wild-type KRAS in the BBP- and four patients in the $\mathrm{BBP}+$ groups underwent cetuximab with or without CPT-11 for subsequent therapies. There was no significant difference in the incidence of KRAS mutation and the induction of subsequent therapies between the BBP- and BBP+ groups. 
Table V. Adverse events.

\begin{tabular}{|c|c|c|c|c|c|c|c|c|c|c|c|c|}
\hline \multirow{2}{*}{$\begin{array}{l}\text { CTCAE } \\
\text { ver. } 3.0 \text { grade }\end{array}$} & \multicolumn{6}{|c|}{ BBP- $(n=27)$} & \multicolumn{6}{|c|}{$\mathrm{BBP}+(\mathrm{n}=15)$} \\
\hline & $1, \mathrm{n}$ & $2, \mathrm{n}$ & $3, \mathrm{n}$ & $4, \mathrm{n}$ & All, $\%$ & $\geq \mathrm{G} 3, \%$ & $1, \mathrm{n}$ & $2, n$ & $3, \mathrm{n}$ & $4, \mathrm{n}$ & All, $\%$ & $\geq \mathrm{G} 3, \%$ \\
\hline Leucopenia & 4 & 2 & 1 & 0 & 25.9 & 3.7 & 1 & 6 & 1 & 1 & 60.0 & 13.3 \\
\hline Neutropenia & 1 & 3 & 2 & 0 & 22.2 & 7.4 & 1 & 6 & 1 & 1 & 60.0 & 13.3 \\
\hline Anemia & 5 & 2 & 3 & 0 & 37.0 & 11.1 & 4 & 1 & 0 & 0 & 33.3 & 0.0 \\
\hline Thrombocytopenia & 4 & 0 & 0 & 0 & 14.8 & 0.0 & 3 & 1 & 0 & 0 & 26.7 & 0.0 \\
\hline Diarrhea & 5 & 8 & 2 & 0 & 55.6 & 7.4 & 5 & 2 & 0 & 0 & 46.7 & 0.0 \\
\hline Anorexia & 3 & 7 & 2 & 0 & 44.4 & 7.4 & 5 & 2 & 0 & 0 & 46.7 & 0.0 \\
\hline Nausea & 5 & 7 & 0 & 0 & 44.4 & 0.0 & 4 & 4 & 0 & 0 & 53.3 & 0.0 \\
\hline Vomiting & 1 & 6 & 0 & 0 & 25.9 & 0.0 & 3 & 0 & 0 & 0 & 20.0 & 0.0 \\
\hline Fatigue & 2 & 4 & 2 & 0 & 29.6 & 7.4 & 3 & 1 & 0 & 0 & 26.7 & 0.0 \\
\hline Stomatitis & 1 & 0 & 0 & 0 & 3.7 & 0.0 & 1 & 0 & 0 & 0 & 6.7 & 0.0 \\
\hline Dizziness & 0 & 0 & 0 & 0 & 0.0 & 0.0 & 0 & 0 & 0 & 0 & 0.0 & 0.0 \\
\hline Neuropathy & 8 & 2 & 0 & 0 & 37.0 & 0.0 & 4 & 1 & 0 & 0 & 33.3 & 0.0 \\
\hline Alopecia & 5 & 5 & 1 & 0 & 40.7 & 3.7 & 3 & 6 & 0 & 0 & 60.0 & 0.0 \\
\hline Hand-foot syndrome & 6 & 1 & 0 & 0 & 25.9 & 0.0 & 3 & 0 & 1 & 0 & 26.7 & 6.7 \\
\hline Proteinuria & 0 & 0 & 0 & 0 & 0.0 & 0.0 & 0 & 0 & 0 & 0 & 0.0 & 0.0 \\
\hline Hypertension & 0 & 1 & 2 & 0 & 11.1 & 7.4 & 2 & 3 & 3 & 0 & 53.3 & 20.0 \\
\hline Bleeding & 0 & 0 & 0 & 0 & 0.0 & 0.0 & 0 & 0 & 0 & 0 & 0.0 & 0.0 \\
\hline
\end{tabular}

BBP, bevacizumab beyond progression; CTCAE, Common Terminology Criteria for Adverse Events.

Table VI. Reasons for treatment discontinuation.

\begin{tabular}{lc}
\hline Reasons for discontinuation & Patients, $\mathrm{n}$ \\
\hline BBP- $\left(\mathrm{n}=24^{\mathrm{a}}\right.$ /27) & \\
Progressive disease & 11 \\
Adverse events & 3 \\
Nausea & 2 \\
Diarrhea & 1 \\
Others & 10 \\
Operation & 3 \\
IC-refusal & 1 \\
PS & 6 \\
BBP+ (n=12 $/ 15)$ & \\
Progressive disease & 9 \\
Adverse events & 3 \\
Nausea & 1 \\
Febrile neutropenia & 1 \\
Allergy & 1 \\
\hline
\end{tabular}

${ }^{a} \mathrm{n}=3 ;{ }^{\mathrm{b}} \mathrm{n}=3$, censored. BBP, bevacizumab beyond progression; IC, informed consent; PS, performance status.

\section{Discussion}

The results showed that the XELIRI regimen plus continuous treatment with bevacizumab was well-tolerated and effective as a second-line chemotherapy beyond disease progression in patients with mCRC. All the adverse events were manageable and all the patients recovered well from these adverse events as a result of reduction or discontinuation of treatment. The XELIRI regimen plus continuous treatment with bevacizumab achieved a DCR of $66.6 \%(C R+P R+S D)$ and led to a significant improvement in OS and PFS as compared with other patients treated without continuous administration of bevacizumab; this was found to be the case regardless of the treatment or response in the first-line setting. Furthermore, 40 patients (95.2\%) including all 15 in the BBP+ and 25 in the BBP-groups, received sequential administration of XELOX and XELIRI and this did not aggravate adverse events indicating that this sequential approach was feasible and manageable for patients with mCRC.

Appropriate schedules and doses regarding the XELIRI regimen have been investigated in a series of clinical trials. The BICC-C study verified the improved safety and efficacy of initial treatment with tri-weekly XELIRI therapy as compared with FOLFIRI and modified IFL therapy. The BICC-C study employed a dose of $250 \mathrm{mg} / \mathrm{m}^{2} \mathrm{CPT}-11$ on day 1 and $2,000 \mathrm{mg} / \mathrm{m}^{2} /$ day capecitabine on days $1-14$ in the XELIRI regimen (4), which raised concerns with regard to gastrointestinal toxicities. Subsequently, an alternative reduced dose was attempted using the XELIRI regimen in the ACCORD 13 study (5). This trial assessed the efficacy and safety of XELIRI and FOLFIRI therapy in combination with bevacizumab as a first-line therapy for patients with mCRC. The ACCORD 13 study employed a reduced dose of $200 \mathrm{mg} / \mathrm{m}^{2}$ CPT-11 on day 1 and $2,000 \mathrm{mg} / \mathrm{m}^{2} /$ day capecitabine on days $1-14$ every 3 weeks in XELIRI regimen and for elderly patients, $\geq 65$ years, a lower daily capecitabine dose $\left(1,600 \mathrm{mg} / \mathrm{m}^{2} / \mathrm{day}\right)$ was administered. This dose modification contributed to reduced gastrointestinal 
toxicities. Consequently, in the present study, the dose in the second-line treatment using the XELIRI regimen was modified according to the ACCORD 13 study, in which a dose of $200 \mathrm{mg} / \mathrm{m}^{2} \mathrm{CPT}-11$ on day 1 and 2,000 (patients aged $<65$ ) or $1,600 \mathrm{mg} / \mathrm{m}^{2} /$ day (patients aged $\geq 65$ years) capecitabine on days 1-14, was administered every 3 weeks. Furthermore, once the dose modification of capecitabine had been carried out in the first-line setting, this reduced dose was also continued in the second-line treatment. These modifications achieved a reduction in the incidence of adverse events.

CTP-11 is converted to its active metabolite, SN-38, by carboxylesterase. SN-38 is then glucuronidated by the enzyme uridine diphosphate glucuronosyltransferase 1A1 (UGT1A1). UGT1A1 has a number of polymorphisms, including UGT1A1*6 and UGT1A1*28 (6-8). Homozygosity or double heterozygosity for the UGT1A1 polymorphisms, UGT1A1*6 and UGT1A1*28, is likely to induce serious adverse events, including neutropenia or diarrhea. A reduced dose of CTP-11 should be required for patients with these polymorphisms (9), but no rules regarding dose modification have been established in clinical studies. At the initiation of the XELIRI regimen, therefore, UGT1A1 genotyping was not explored in our hospital. The patient who developed grade 4 neutropenia did not receive a reduced dose of CTP-11 and was later identified as being double heterozygous for UGT1A1*6 and UGT1A1*28. In order to prevent severe adverse events associated with the metabolism of CTP-11, UGT1A1 genotyping should be checked and patients who are homozygous or double heterozygous for UGT1A1*28 and UGT1A1*6 should be administered a reduced dose of CTP-11.

In second-line treatment, there have been several phase II studies that have assessed the XELIRI regimen in patients previously treated with oxaliplatin. ML18147 was a phase III study that demonstrated the benefits of continued use of bevacizumab plus standard second-line chemotherapy following standard first-line bevacizumab-based treatment (3). The efficacy analysis revealed a median PFS time of 5.7 months. This study employed the tri-weekly XELIRI plus bevacizumab regimen used in the AIO trial 0604 (10) in $12 \%$ of the total patient population. The present study achieved a median PFS time of 7.2 months using a tri-weekly XELIRI plus bevacizumab regimen; this indicated that the XELIRI regimen in combination with bevacizumab was effective in a second-line setting. In addition, patients who were less likely to respond to first-line treatment, such as those with a first-line PFS time of $<3$ months, were excluded from the ML18147 trial. The results showed that no difference in second-line PFS time was found between patients with poor response in the first-line setting and other patients, as long as there was continuous administration of bevacizumab in the second-line setting, indicating that the XELIRI regimen in combination with bevacizumab in a second-line setting provided a clinical benefit for those patients who were less likely to respond to the first-line treatment.

The BIX study was conducted to verify this tri-weekly XELIRI plus bevacizumab regimen $(7.5 \mathrm{mg} / \mathrm{kg}$ bevacizumab on day $1,200 \mathrm{mg} / \mathrm{m}^{2} \mathrm{CPT}-11$ on day 1 and $1,600 \mathrm{mg} / \mathrm{m}^{2} /$ day capecitabine on days 1-14) in Japanese patients with mCRC who had been previously treated with oxaliplatin and bevacizumab (11). The BIX study showed that the most common grade $3 / 4$ adverse events were nausea (5.9\%), diarrhea (5.9\%), fatigue (2.9\%) and neutropenia (8.8\%). The efficacy analysis revealed an overall response rate of $17.6 \%$ and a PFS time of 8.3 months. These data were consistent with those in the present study, indicating that XELIRI plus bevacizumab can be expected to be safe and effective for Asian patients in a second-line setting.

In conclusion, the present study results should be interpreted within the context of the study limitations and more studies, including multinational randomized phase III studies, are required to draw definitive conclusions. However, we believe that the XELIRI regimen with continuous treatment of bevacizumab could be well-tolerated and effective as a second-line chemotherapy beyond disease progression in patients with $\mathrm{mCRC}$.

\section{Acknowledgements}

The present study was supported in part by a grant-in-aid of the postgraduate students from Jichi Medical University, a grant-in-aid from the Ministry of Education, Culture, Sports, Science and Technology and the JKA Foundation through its promotion funds from Keirin Racin.

\section{References}

1. Saltz LB, Clarke S, Díaz-Rubio E, et al: Bevacizumab in combination with oxaliplatin-based chemotherapy as first-line therapy in metastatic colorectal cancer: a randomized phase III study. J Clin Oncol 26: 2013-2019, 2008.

2. Hochster HS, Hart LL, Ramanathan RK, et al: Safety and efficacy of oxaliplatin and fluoropyrimidine regimens with or without bevacizumab as first-line treatment of metastatic colorectal cancer: results of the TREE Study. J Clin Oncol 26: 3523-3529, 2008.

3. Bennouna J, Sastre J, Arnold D, et al; ML18147 Study Investigators: Continuation of bevacizumab after first progression in metastatic colorectal cancer (ML18147): a randomised phase 3 trial. Lancet Oncol 14: 29-37, 2013.

4. Fuchs CS, Marshall J, Mitchell E, et al: Randomized, controlled trial of irinotecan plus infusional, bolus, or oral fluoropyrimidines in first-line treatment of metastatic colorectal cancer: results from the BICC-C Study. J Clin Oncol 25: 4779-4786, 2007.

5. Ducreux M, Adenis A, Pignon JP, et al: Efficacy and safety of bevacizumab-based combination regimens in patients with previously untreated metastatic colorectal cancer: final results from a randomised phase II study of bevacizumab plus 5-fluorouracil, leucovorin plus irinotecan versus bevacizumab plus capecitabine plus irinotecan (FNCLCC ACCORD 13/0503 study). Eur J Cancer 49: 1236-1245, 2013.

6. Ando Y, Saka H, Ando M, et al: Polymorphisms of UDP-glucuronosyltransferase gene and irinotecan toxicity: a pharmacogenetic analysis. Cancer Res 60: 6921-6926, 2000.

7. Innocenti $\mathrm{F}$, Undevia SD, Iyer L, et al: Genetic variants in the UDP-glucuronosyltransferase 1A1 gene predict the risk of severe neutropenia of irinotecan. J Clin Oncol 22: 1382-1388, 2004.

8. Minami H, Sai K, Saeki M, et al: Irinotecan pharmacokinetics/pharmacodynamics and UGT1A genetic polymorphisms in Japanese: roles of UGT1A1*6 and *28. Pharmacogenet Genomics 17: 497-504, 2007.

9. O'Dwyer PJ and Catalano RB: Uridine diphosphate glucuronosyltransferase (UGT) 1A1 and irinotecan: practical pharmacogenomics arrives in cancer therapy. J Clin Oncol 24: 4534-4538, 2006.

10. Schmiegel W, Reinacher-Schick A, Arnold D, et al: Capecitabine/irinotecan or capecitabine/oxaliplatin in combination with bevacizumab is effective and safe as first-line therapy for metastatic colorectal cancer: a randomized phase II study of the AIO colorectal study group. Ann Oncol 24: 1580-1587, 2013.

11. Yamaguchi T, Hamamoto $\mathrm{Y}$ and $\mathrm{T} \mathrm{N}$ : A Phase I/II study of tri-weekly XELIRI plus bevacizumab for Japanese patients with mCRC as second-line chemotherapy (BIX study). Ann Oncol 23 abstr P-0245, 2012. 